\title{
Chemical Sensors Based on Metal Oxide Nanostructures
}

\author{
Gary W. Hunter ${ }^{\mathrm{a}}$, Jennifer C. Xu ${ }^{\mathrm{a}}$, Laura J. Evans ${ }^{\mathrm{a}}$, Randy L. Vander Wal ${ }^{\mathrm{b}}$, Gordon M. \\ Berger ${ }^{\mathrm{b}}$, Mike J. Kulis ${ }^{\mathrm{b}}$, and Chung-Chiun Liu ${ }^{\mathrm{c}}$
}

a) NASA Glenn Research Center Cleveland, $\mathrm{OH} 44135$

b) USRA at NASA Glenn Research Center, Cleveland, OH 44135

c) Case Western Reserve University, Cleveland, OH 44106

This paper is an overview of sensor development based on metal oxide nanostructures. While nanostructures such as nanorods show significant potential as enabling materials for chemical sensors, a number of significant technical challenges remain. The major issues addressed in this work revolve around the ability to make workable sensors. This paper discusses efforts to address three technical barriers related to the application of nanostructures into sensor systems: 1) Improving contact of the nanostructured materials with electrodes in a microsensor structure; 2) Controling nanostructure crystallinity to allow control of the detection mechanism; and 3) Widening the range of gases that can be detected by using different nanostructured materials. It is concluded that while this work demonstrates useful tools for further development, these are just the beginning steps towards realization of repeatable, controlled sensor systems using oxide based nanostructures.

\section{Introduction}

Metal-oxide semiconductors, such as semiconducting tin oxide $\left(\mathrm{SnO}_{2}\right)$, have been used as chemical sensors for a number of years. Applications include environmental monitoring, fire detection, and vehicle monitoring. The fundamental sensing mechanism of these metal oxide based gas sensors relies upon the change in electrical conductivity due to the interaction between the gases in the environment and oxygen in the grain boundaries. Drift in the properties of $\mathrm{SnO}_{2}$ with long term heating due to grain boundary annealing have been previously noted $(1,2)$. In order to stabilize the $\mathrm{SnO}_{2}$ grain structure for long-term operation, the fabrication of nanocrystalline $\mathrm{SnO}_{2}$ has been investigated. Nanocrystalline materials have several inherent advantages over conventionally fabricated materials including increased stability and sensitivity at high temperatures $(3,4] \backslash)$. Further, a nanocrystalline $\mathrm{SnO}_{2}$ based $\mathrm{NO}_{\mathrm{x}}$ sensor has been tested in a power generation turbine engine (5) as well as part of an array. Nanocrystalline materials provide a significant increase in the surface/volume ratio for a material. Given that the response for this type of sensor is dependent on grain boundaries, this results in more sensitive sensors that can operate at lower temperatures. More importantly, the reaction mechanisms which govern the sintering process are different for nanocrystalline materials than bulk or macrograined material. In particular, the grains are more stable and less likely to sinter, yielding a more stable sensor. Therefore, the use of nanocrystalline material results in a decrease of grain growth while improving sensor sensitivity, stability, and response time. 
Thus, while layers of nanocrystals have shown significant potential for chemical sensing applications, the advantages of nanostructured oxide sensors, e.g. nanorods, nanofibers, nanoribbons, nanotubes, and nanobelts, are just beginning to be explored. Given the nanocrystalline example, it is believed that significant gains in sensor performance can be achieved by use of these nanostructures. However, these gains must be demonstrated, and significant technical challenges remain before routine implementation of nanostructured oxides in sensing applications can take place. In the end, it is irrelevant to a user that a sensor is based on nanotechnology; what will allow nanosensor system application are improved capabilities compared to standard micro or macro sensor technology. This includes sensor reproducibility, sensitivity, selectivity, stability, and response time.

This paper gives a brief overview of sensor development which focuses on moving from nanocrystalline materials to nanostructures as the active sensing material. Issues addressed include: fabrication approaches to metal oxide nanostructures, integration of metal oxide nanostructures into sensor microstructures, control of nanostructure crystallinity to allow control of the detection mechanism, and the fabrication of a range of oxide materials from tin oxide to iron oxide. It is concluded that while this work demonstrates useful tools for further development, these are the beginning steps towards realization of repeatable, controlled sensor systems using metal oxide based nanostructures.

\section{Oxide Nanostructure Fabrication Approaches}

Several different methods have been studied for fabrication of the oxide nanostructures in this work. The approach is to have a range of tools available to control the nanostructures produced. Some processes produce material which is later transferred to a sensor microstructure while in other processes the nanostructures are grown directly on the sensor platform. The paragraphs below in this section describe the nanostructure fabrication approaches while the next section describes integration of the oxide nanostructures into microsensor structures as well as other sensor development topics.

\section{Thermal Evaporation-Condensation (TEC)}

Thermal evaporation-condensation (TEC) can be used to create a range of metal oxide nanostructures. The corresponding metal is evaporated within the flow of an inert gas plus a minute amount of oxygen. For example, to produce tin dioxide, tin powder is held within an alumina boat and evaporated species form nanorods within the same boat, along the boat edges, and on the surface of the source material. Growth occurs via a vapor-solid (VS) mechanism in the absence of catalysts, although an oxide assisted mechanism may also contribute. Nanoforms are collected downstream from plates positioned at lower temperature regions. Nanorods, nanoblades, or tetrapods may be formed depending upon the details of the furnace temperature, gas flow rate, and temperature of the collection zone. Typical processing conditions include a temperature of $\sim 1000^{\circ} \mathrm{C}$ and flow of an inert gas such as argon. Similar TEC approaches are used to fabricate other oxides. 


\section{Electrospinning}

Electrospinning is used to fabricate polycrystalline nanofibers. Since the electrospinning process can be computer-controlled, this approach can yield more repeatable deposition of sensing material. Electrospinning is a process whereby a charged, viscous solution is drawn from a capillary and retains a "threadlike" form while depositing upon a grounded surface. In the electrospinning process, a mixture of metal alkoxide and polymer is used as the precursor mixture. By incorporating different metal alkoxide precursors into the solution, different oxide materials can be produced in the form of nanofibers. These solutions are fed by a syringe pump to an electrified 22 gauge needle from which a filament emerges under the action of high negative voltage $(15-20$ $\mathrm{keV}$ ) between the needle and the ground. As the fiber traverses the distance between the needle and substrate, the solvent evaporates, yielding a semi-solid nanofiber. Upon calcination, the polymer is oxidized and the resulting sol gel is solidified to form a metal oxide, polycrystalline nanofiber. This yields a 1-D sensor element as compared with the 1-D single crystal nanorods as formed by the thermal evaporation-condensation approach described previously.

\section{Flame Deposition}

Flame deposition is a method by which a metal alkoxide or metal salt is dissolved in an alcohol carrier fluid. This mixture is atomized using a spray jet nebulizer. The resulting mist is carried into a gas-jet diffusion flame with surrounding air co-flow. Within the flame, the metal precursor decomposes with subsequent oxidation of the metal atoms and clusters. Thermophoretic forces are exploited to deposit the resulting fine metal oxide aerosol as a film of oxide nanostructures. As deposited, the oxide nanostructures are crystalline and resistant to sintering by virtue of the flame process.

\section{Sensor Development}

\section{Micro-Nano Contact Formation}

One major issue associated with sensor fabrication using oxide nanostructures is the integration of the nanostructured oxides into the sensor structures. In effect, no matter how good the sensing material, if one cannot make contact with it or implement it in a sensor structure, its sensing applicability is limited. Thus, in order to control nanotechnology, it is suggested that one first has to have some control of microtechnology to interface it with the nanostructures. This work is based on a significant history of implementing MEMS (Micro ElectroMechanical Systems) approaches in the development of chemical sensors [6,7]. The MEMS structures themselves will be modified in order to assist in the alignment of the nanostructures, vary sensor response, produce sensor arrays, and fabricate operational systems.

Examples of initial efforts to fabricate oxide nanostructured sensors on microsensor platforms are shown in Figure 1. Tin oxide nanorods produced in large quantities using the TEC process described in the previous section can result in nanostructures as shown in Figure 1a. One approach towards integrating these nanostructures into a sensor involved manipulation of these nanorods by hand onto a substrate, followed by bonding with silver epoxy to make contact with the sensor material as shown in Figure 1b. A 
second approach was to produce a nanorod powder which was put into a solution and precipitated on the substrate between two electrodes as shown in Figure 1c. Both approaches produced sensors which showed responses to hydrogen at $450^{\circ} \mathrm{C}$ (not shown). However, because the density of the nanorod materials, quality of the contact, and variation of baseline material properties were difficult to control, these approaches had limited reproducibility.

a)

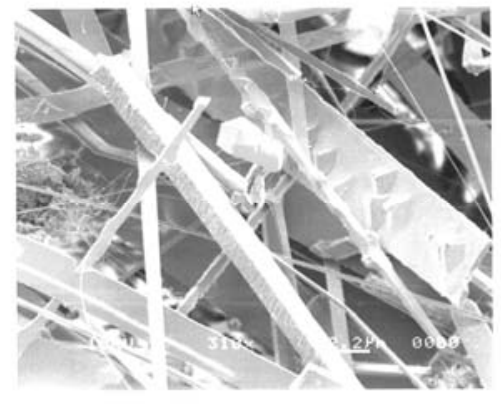

b)

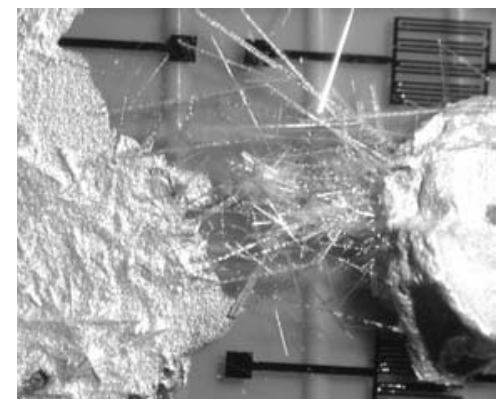

c)

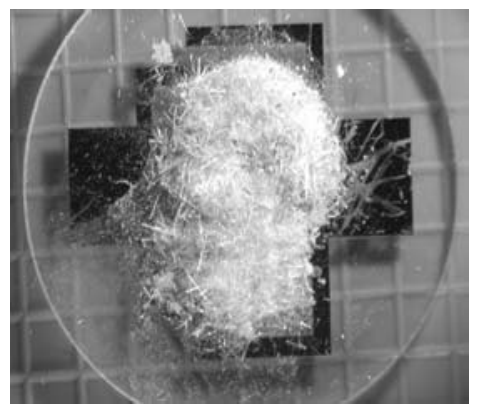

Figure 1. a) Tin oxide nanorods produced using the thermal evaporation-condensation process, b) the nanorods contacted with the substrate via a silver epoxy, c) the nanorods precipitated onto the substrate between two electrodes.

These reproducibility issues have led to an investigation of alternate approaches to the deposition of nanostructures with the objective of controlling the location and density of the nanostructures placed in sensor structures. Three general methods have been investigated using oxide nanostructures on MEMS structures in this work: in-situ chemical vapor deposition (CVD), electrospinning, and alignment of TEC grown nanostructures by methods such as dielectrophoresis. The in-situ CVD growth involves forming MEMS structures, e.g., a gap etched between electrodes, and growing nanotubes across the gap. This approach is discussed in reference 8 for carbon nanotubes and will not be discussed here.

The basic concept behind the second approach, electrospinning, was described earlier in the previous section. Typical distances between the needle and the sensor pattern, which served as the electrical ground, range between 15 and $30 \mathrm{~cm}$. A significant feature of electrospinning is that a linear 1-D nanofilament is formed during the deposition process. Given the charged nature of the polymer solution, the nanofilament has a tendency to repel itself. This feature, combined with the formation of an image charge upon the electrode in contact with the filament, aids in the alignment of the fibers as roughly parallel strands connecting the opposing electrical contacts. These nanofibers may be fabricated upon a variety of sensor configurations and can serve to provide the environmentally sensitive conductive bridge between interdigitated electrodes. This "bridging" has led to pursuit of a sensor construction based on this "telegraph" configuration, where the nanofibers bridge adjacent electrodes.

Figure 2a shows a picture of electrospun $\mathrm{SnO}_{2}$ with a limited doping of palladium (Pd) across two platinum (Pt) electrodes of a MEMS interdigitated finger structure. The Pd doping is achieved by first electrospinning the sample across the interdigitated fingers and then sputtering a very thin layer of Pd catalyst (flash sputter). The Pd thickness is too thin to form a connection between the electrodes but enough to deposit dispersed islands 
of $\mathrm{Pd}$ onto the nanofibers. Figure $2 \mathrm{~b}$ shows the response of the sensor at room temperature as the sensor is exposed first to air, then nitrogen and then a mixture of $0.5 \%$ $\mathrm{H}_{2}$ in nitrogen or $0.5 \%$ methane in nitrogen in the time sequences shown in the figure. The gas flow is controlled so that there no humidity introduced into the gas sample flow during any of the testing; possible humidity effects will be addressed in future studies. A basic description of the testing system is given in reference 9. The sensor has minimal response to changes in air, nitrogen, or methane but a notable response to hydrogen at room temperature. Reference samples with the same interdigitated fingers and with flash sputtered Pd catalysts, but without the electrospun $\mathrm{SnO}_{2}$ were also fabricated and tested. No sensor response was observed for any of the gases. This data shows the capability of electrospun doped $\mathrm{SnO}_{2}$ to form a sensing structure with detection capabilities at room temperature.

a)

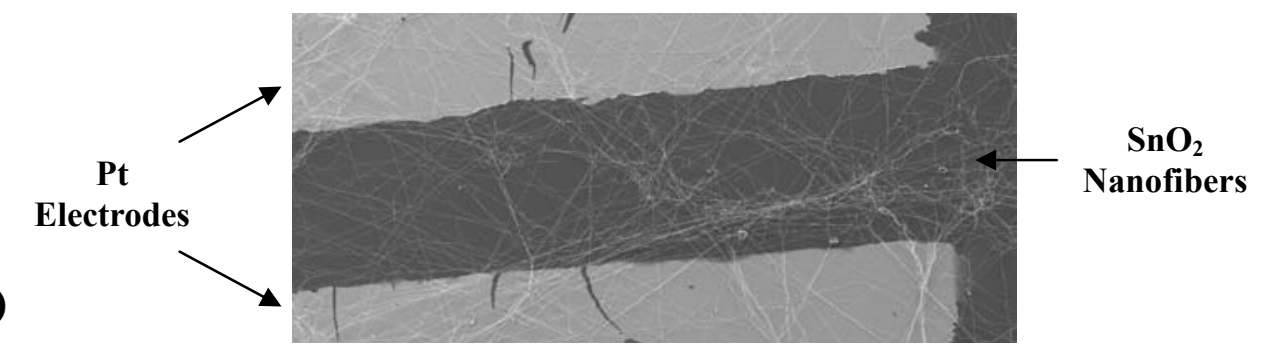

b)

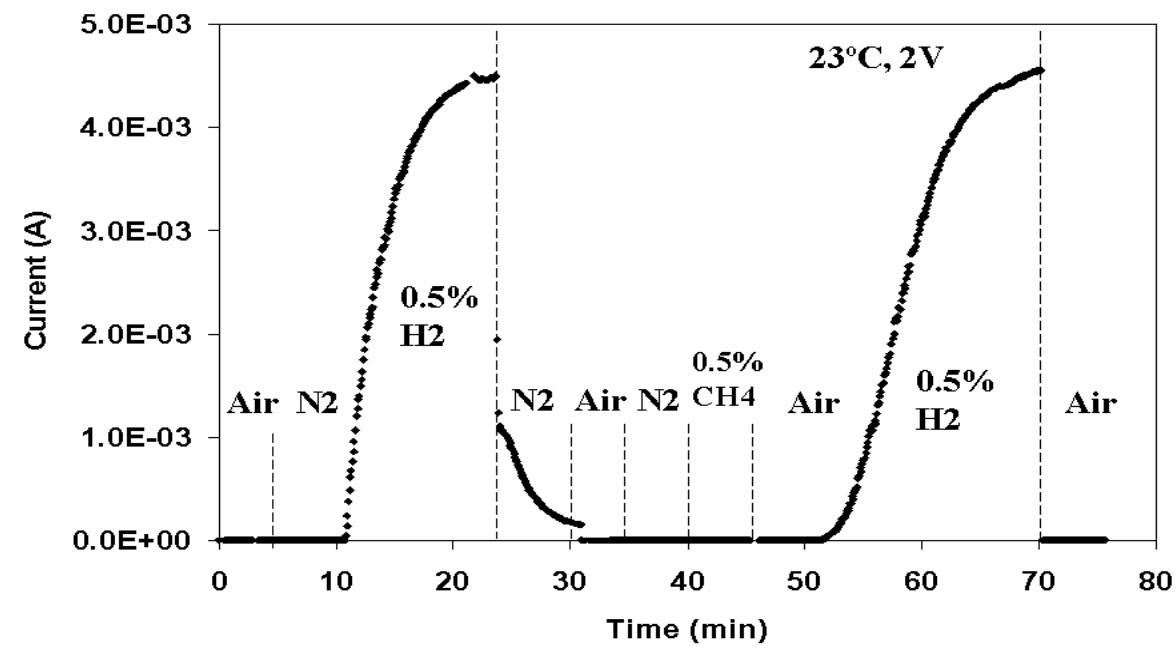

Figure 2. a) Bridging of electrospun $\mathrm{SnO}_{2}$ nanofibers across electrodes. b) Current response at $2 \mathrm{~V}$ of electrospun $\mathrm{Pd}$ doped $\mathrm{SnO}_{2}$ nanofibers to hydrogen and methane in nitrogen at room temperature.

The third approach to align the nanorods so as to bridge MEMS electrode structures is dielectrophoresis. Dielectrophoresis (dielectric field differences) exploits the dielectric difference between the solvent and the nanostructures in the solvent to induce temporary dipoles that align with the imposed electric field. In this process, an AC voltage is applied across the electrode grid using a function generator. For nanorods less than 10 microns long, $10 \mathrm{~V} \mathrm{AC}$ at a frequency of $5 \mathrm{MHz}$ is applied. For nanowires greater than 10 microns, a lower frequency appears to improve alignment. For example, lowering the frequency from $5 \mathrm{MHz}$ to $500 \mathrm{KHz}$ appeared to improve the alignment of $\mathrm{SnO}_{2}$ nanowires that had a length greater than 20 microns long. The solvent is allowed to 
evaporate with the voltage applied to the grid. The resistance across the grid is measured after the solvent completely evaporates. Typically a measurable resistance (less than 40 $\mathrm{M} \Omega$ ) is found after 4 drop/evaporation cycles are completed. After each deposition step, the nanowire placement on the interdigitated grid is observed using an optical microscope to verify deposition uniformity of nanorods.
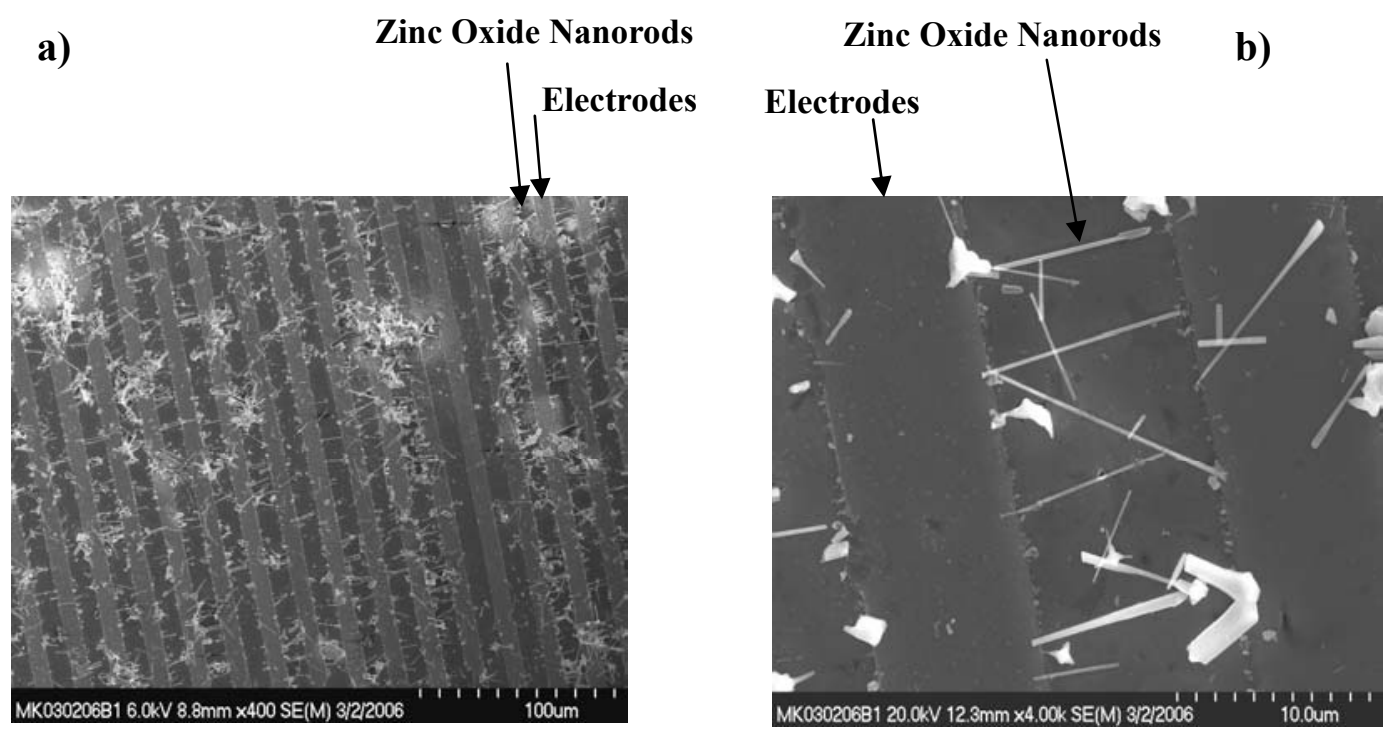

Figure 3. a) Zinc oxide nanorods after dielectrophoresis across a pattern of interdigitated fingers and $b$ ) a magnified view showing parallel alignment of the nanorods across the electrodes.

Figure 3 shows the results of the use of dielectrophoresis to align nanorods across patterned electrodes. Figure 3a shows zinc oxide nanorods after dielectrophoresis across a pattern of interdigitated fingers. The net result is a significant alignment of the nanorods across the patterned electrodes. Figure $3 \mathrm{~b}$ shows a magnified view of these nanorods showing parallel alignment of individual nanorods in some regions. Other regions showed less parallel alignment, in part due to the density of the applied nanostructures. While further refinement of this approach is necessary, this demonstrates the basic capability to align nanostructures with sensing electrodes using dielectrophoresis. Future work will be to improve the uniformity of the nanostructure alignment across the whole electrode pattern and to characterize sensors produced by this technique.

\section{$\underline{\text { Nanomaterial Structure Control }}$}

Metal oxide semiconductors standardly function as sensors by reactive adsorption at oxygen sites, vacancies, or other surface defects. However, the reaction mechanism for nanostructures and the dependence of that mechanism on defects in the nanostructures is not clearly understood. Controlling the nanostructure should improve the control of how many defects are in the material. Using different fabrication techniques and combinations, we are investigating the level of defect structure within the nanoelements used for sensing. The synthesis capability now extends from single crystalline nanowires and nanoribbons (produced through TEC techniques) to polycrystalline nanofibers (produced via electrospinning of sol-gel solutions). Starting from these two extremes, we 
are seeking to tailor the sensing element nanostructure to achieve optimal selectivity and sensitivity. The approach is to control the level of defect structure within the nanoelements used for sensing by using different fabrication techniques.
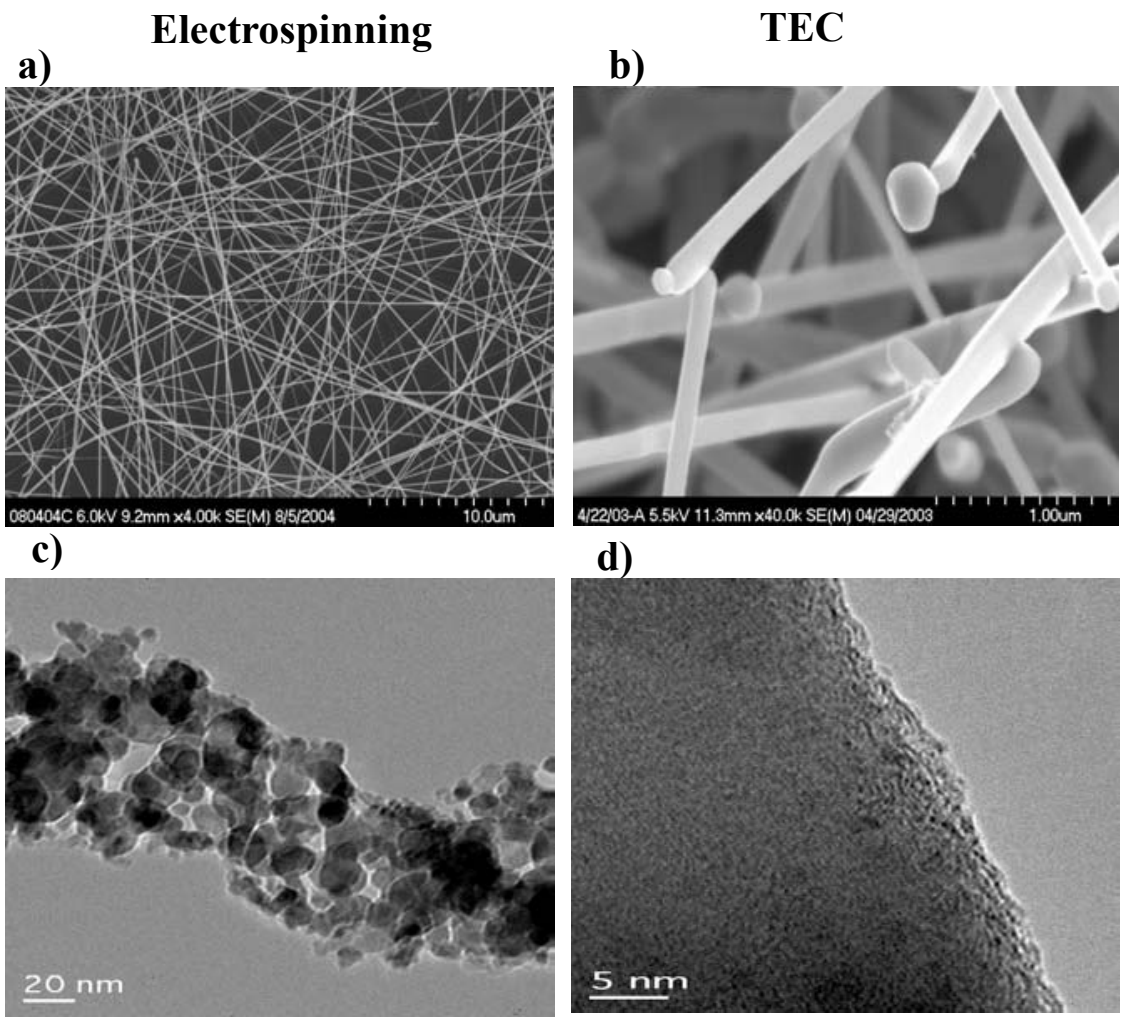

Figure 4. Control of $\mathrm{SnO}_{2}$ nanostructure fabrication: SEM of a) electrospun nanofibers, b) TEC grown nanorods; and HRTEM of c) electrospun nanofibers and d) TEC nanorods. The different processing techniques result in very different crystal structures.

For example, Figure 4 demonstrates the ability to control grain size by the production of single crystalline nanorods and polycrystalline nanofibers of $\mathrm{SnO}_{2}$. The SEM pictures in Figure $4 \mathrm{a}$ and $\mathrm{b}$ show basic differences in structure of the $\mathrm{SnO}_{2}$ formed by the two techniques. The electrospun nanofibers are continuous in structure forming an almost weblike formation as deposited. In contrast, the TEC fabricated nanorods have distinct shapes and structures with possible variance even broader than that shown in Figure $4 \mathrm{~b}$. Closer examination of the crystal microstructure resulting from the two deposition techniques is shown in Figure $4 \mathrm{c}$ and d. While at slightly different magnifications, the two high resolution TEM pictures clearly show a difference in microstructure between the two $\mathrm{SnO}_{2}$ samples produced by TEC and electrospinning. While the TEC produced device is a single crystal, the electrospun system is composed of multiple grains. Work is on-going to correlate the grain properties of the $\mathrm{SnO}_{2}$ to the sensor material response.

\section{Other Nano Oxide Materials}

Significant progress has been made in varying the range of nanostructures available for sensing applications. The approach is to have a variety of possible materials available to detect a range of species in various environments. This approach reflects activities in 
microsensor development which employs multiple materials and platforms to interrogate the environment (7). The range of nanostructured materials fabricated now includes zinc oxide $(\mathrm{ZnO})$, indium oxide $\left(\mathrm{In}_{2} \mathrm{O}_{3}\right)$, and iron oxide $\left(\mathrm{Fe}_{2} \mathrm{O}_{3}\right)$. As with $\mathrm{SnO}_{2}$, the capability to fabricate these nanostructures either through TEC or electrospinning techniques has been investigated. Figure 5 shows $\mathrm{ZnO}$ and $\operatorname{In}_{2} \mathrm{O}_{3}$ fabricated using both techniques; as with $\mathrm{SnO}_{2}$, examination of the resulting material suggests that the deposition pattern and crystal properties vary significantly with deposition technique.

a)

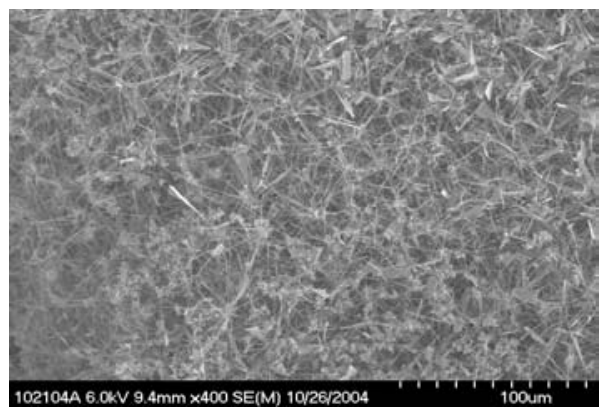

c)

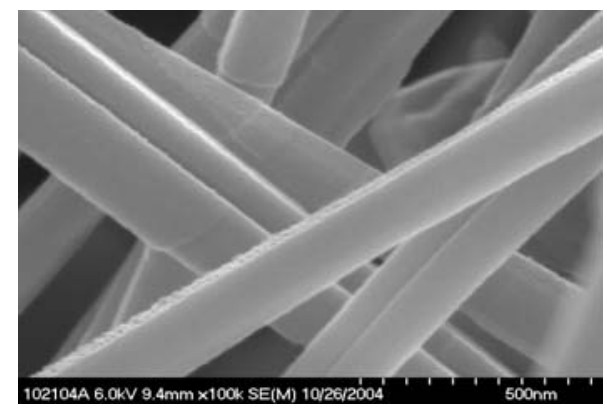

b)

$$
\operatorname{In}_{2} \mathbf{O}_{3}
$$

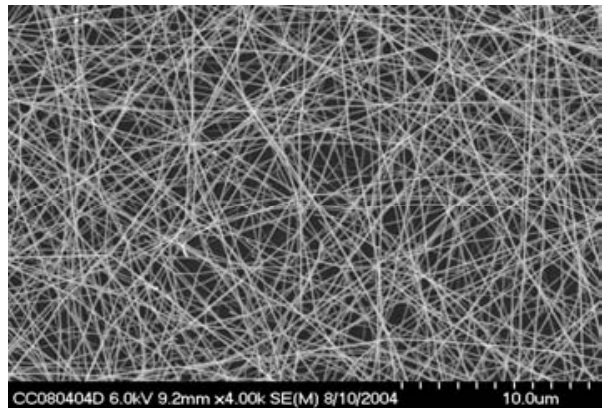

d)

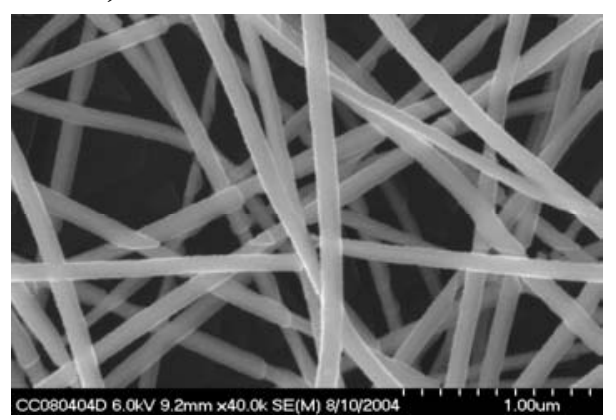

Figure 5. Oxide nanostructures formed from electrospinning (nanofibers) and TEC (nanorods): a) $\mathrm{ZnO}$ nanofibers, b) $\mathrm{In}_{2} \mathrm{O}_{3}$ nanofibers, c) $\mathrm{ZnO}$ nanorods, d) $\mathrm{In}_{2} \mathrm{O}_{3}$ nanorods.

As noted above, catalyst coatings allowed room temperature detection of hydrogen with a Pd coated $\mathrm{SnO}_{2}$ electrospun system. Coating of other materials such as zinc oxide $(\mathrm{ZnO})$ has been accomplished with such catalysts as $\mathrm{Pd}$, gold $(\mathrm{Au})$, and copper $(\mathrm{Cu})$. For example, Figure 6 shows the response at room temperature of a sensor composed of a catalyst coated $\mathrm{ZnO}$ placed by dielectrophoresis on interdigitated fingers as the sensor is exposed first to air, nitrogen, and then a mixture of $0.5 \% \mathrm{H}_{2}$ in nitrogen in the time sequences shown in the figure. The sensor has minimal response to changes in air or nitrogen, but does respond to hydrogen in nitrogen at room temperature. This is consistent with the capabilities of oxide nanostructures with a catalyst coating to detect hydrogen and confirms that these capabilities extend to materials beyond $\mathrm{SnO}_{2}$. Future work will include investigating the detection capabilities of various material types and tailoring the material for the application.

The possible applications of oxide nanostructures also include detection of gases at higher temperatures, which might be of interest for emission monitoring applications (5, 7). Figure 7 shows the response of $\mathrm{Fe}_{2} \mathrm{O}_{3}$, fabricated by flame deposition as described in the processing section, on interdigitated fingers without doping to $0.5 \%$ propylene in nitrogen at $485^{\circ} \mathrm{C}$ in a test procedure composed of sequences of air, $\mathrm{N}_{2}$, and propylene. $\mathrm{A}$ 
response of several orders of magnitude is observed upon exposure to propylene with a quick subsequent recovery to baseline upon exposure to air. This highly sensitive response shows the potential of oxide nanostructures to detect hydrocarbon species at higher temperatures.

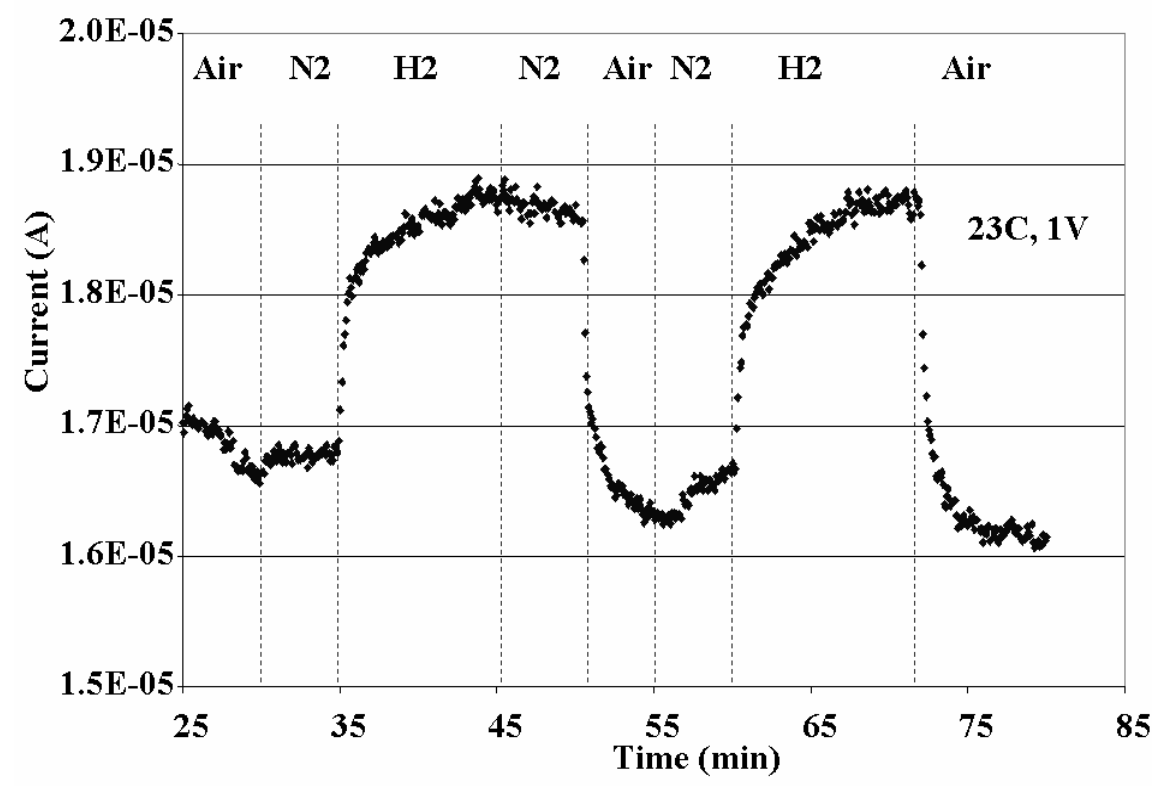

Figure 6. Response of $\mathrm{Pd}$ catalyst doped $\mathrm{ZnO}$ sensor to cycles of air, nitrogen, and $0.5 \%$ hydrogen in nitrogen showing gas sensing capabilities at room temperature.

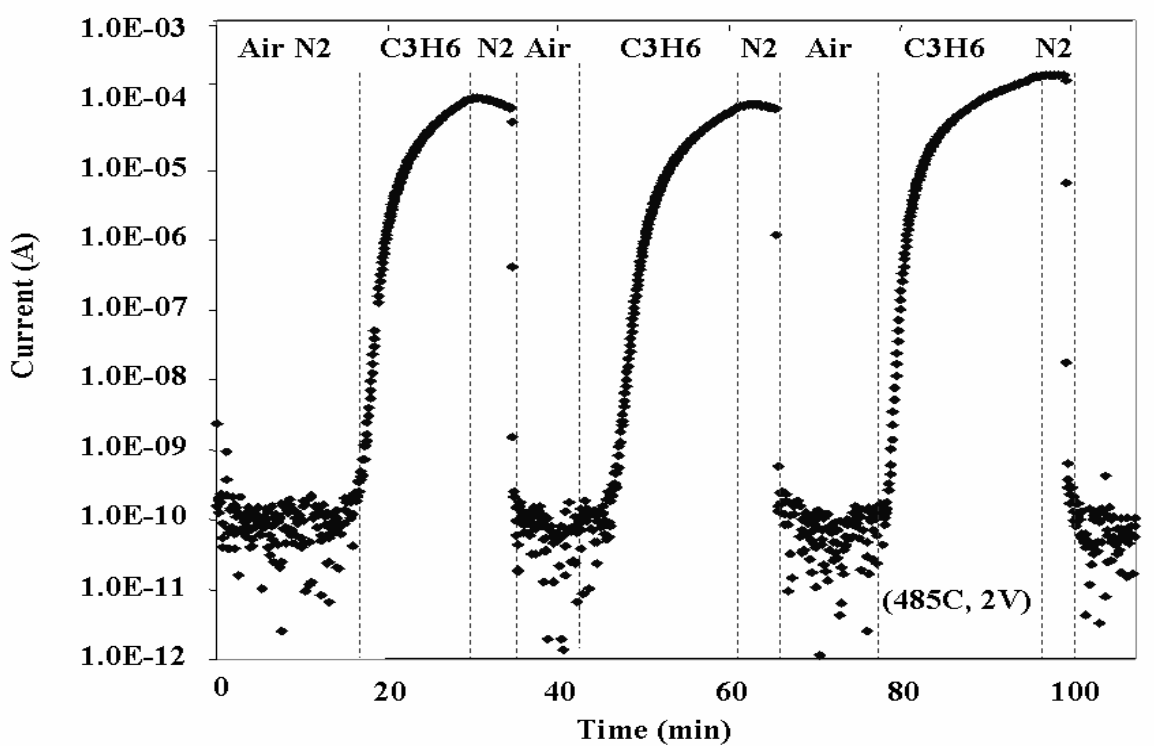

Figure 7. Response of $\mathrm{Fe}_{2} \mathrm{O}_{3}$ sensor to cycles of air, nitrogen, and $0.5 \%$ propylene in nitrogen at $485^{\circ} \mathrm{C}$. The ability of the sensor to respond to propylene with high sensitivity is shown.

\section{Conclusions}

This paper presents an overview of sensor development based on metal oxide structures with the objective of enabling the fabrication of stable, reproducible sensors 
with improved capabilities over standard technology. This paper addresses three technical barriers related to the application of nanostructures into sensor systems: 1) improving contact of the nanostructured materials with electrodes in a microsensor structure; 2) controlling nanostructure crystallinity to allow control of the detection mechanism; and 3 ) widening the range of gases that can be detected by using different nanostructured materials.

Overall, this work demonstrates useful tools for further development and the ability to form operational sensors at both room temperature and at higher temperature has been shown. However, processing control and optimization of the sensors for a given set of gases and operational environments is not yet mature. Significant further work is necessary towards the realization of repeatable, controlled sensor systems composed of

oxide based nanostructures.

\section{Acknowledgements}

We gratefully acknowledge the contributions of those who made this paper and its contents possible: Dr. L. Matus, Dr. M. Zeller, Dr. Carl Chang, John Wrbanek, and Dr. G. Beheim, and T. Guo of NASA GRC, D. Androjna, M. Artale, P. Lampard of Sierra Lobo/NASA GRC; Dr. J. Stetter of SRI; and Prof. Thomas M. Ticich, Centenary College of Louisiana, Shreveport, Louisiana.

\section{References}

1. H. Ogawa, M. Nishikawa, and A. Abe, Journal of Applied Physics, 53, 4448-4455 (1988).

2. C. Xu, J. Tamaki, N. Miura, and N. Yamazoe, Sensors and Actuators, B, 3, 147-155 (1991).

3. D. J. Yoo, J. Tamaki, S. J. Park, N. Miura, and N. Yamazoe, Journal of Electrochemical Society, 142, L105-L107 (1995).

4. R. Vogel, P. Hoyer, and H. Weller, Journal of Physical Chemistry, 98, 3183-3188 (1994).

5. G. W. Hunter, P. G. Neudeck, G. Fralick, C. C. Liu, Q. H. Wu, S. Sawayda, Z. Jin, D. B. Makel, M. Liu, W. A. Rauch, and G. Hall, Microfabricated Systems and MEMS IV/2000, P.J. Hesketh, S. S. Ang, W. E. Bailey, J. L. Davidson, H. G. Hughes, and D. Misra, Editors, PV 2000-19, p. 126, The Electrochemical Society Proceedings Series, Phoenix, AZ, (2000).

6. C. C. Liu, P. K. Hesketh, and G. W. Hunter, The Electrochemical Society Interface, 13, 22-29 (2004).

7. G. W. Hunter, J. C. Xu, C. C. Liu, and D. B. Makel, in The MEMS Handbook Second Edition: Design and Fabrication, M. Gad-El-Hak, Editor, Chapter 11, CRC Press, Baton Rouge (2006).

8. W. J. Buttner, G.H. Chung, L. Evans, G. Hunter, J. R. Stetter, J.S. Jeong, J. Xu, R. Wang, and R. Vander Wal, "Surface Characterization and Manipulation of SWCNT for Sensor Applications", ECS Trans. 1, 9, (2006).

9. G.W. Hunter, L. Chen, P.G. Neudeck, C.C. Liu, Q.H. Wu, and H.J. Zhou, "Developments in High Temperature Gas Sensor Technology" 42nd Turbo-Expo 
'97, International Gas Turbine Institute of the American Society of Mechanical Engineers Orlando, Florida, June, 1997, NASA TM 107442. 